\title{
LabJack-like crystal structures of halogenated 2-phenylbenzimidazoles - are they isostructural?
}

\author{
P Bombicz ${ }^{1}, \mathrm{~N} \mathrm{May}^{2}$, D Fegyverneki ${ }^{2}$, A Saranchimeg ${ }^{2}$, G Szabó $^{2}$, Z Makó $^{2}$, G Gál $^{2}$, L Bereczki ${ }^{2}$ \\ ${ }^{1}$ Research Centre for Natural Sciences, Budapest, ${ }^{2}$ Research Centre for Natural Sciences, \\ Budapest, Hungary \\ bombicz.petra@ttk.mta.hu
}

Investigation of isostructurality is a tool of understanding of the close packing principles. Isostructurality calculations and statistical analyses are efficient tools for finding isostructural crystals. The fine tuning of structural properties can be achieved by application of substituents, changing its placement and/or chemical composition influencing electrostatic and spherical properties. Molecular conformation of flexible molecules may adjust to the supramolecular features. Crystal structures of 2-phenylbenzimidazole derivatives were analysed, either prepared by covalent synthesis or collected from the CSD. The molecules are substituted on the phenyl ring in ortho, meta and para positions or simultaneously in two different positions with fluorine, chlorine and bromine. The arrangement of the molecules recalls a laboratory Jack of which the height is determined by the halogen substitution. The molecular arrangements in the crystals are governed by the N-H...N intermolecular interaction. The relative tilt of the hydrogen bonded molecules is determined by the substituents of the 2-phenylbenzimidazole skeleton. We focus on how and to what extent the type and the position of a halogen substituent may affect the crystal structure. We compare a series of increasingly more different structures to test the limits of isostructurality. With the varying substitution the lengths of the unit cell axes, the tilt of the molecules and the supramolecular interactions can be systematically influenced "the lab Jack can be closed or opened". The flexibility of the structures permits a nearly twofold increase of the length of the unit cell perpendicular to the N-H...N hydrogen bonded chains in the investigated crystals with unchanged space group and $\mathrm{Z}$. How far can we call these crystals being isostructural? Figure 1 Superimposed unit cells of the investigated crystals indicating the arrangement of the molecules. This work was supported by the National Research, Development and Innovation Office-NKFIH through OTKA K124544 and KH129588. 1. Petra Bombicz, Nóra V. May, Dániel Fegyverneki, Avirmed Saranchimeg, Laura Bereczki: Methods for easy recognition of isostructurality - Lab Jack-likecrystal structures of halogenated 2phenylbenzimidazoles. CrystEngComm DOI: 10.1039/D0CE00410C

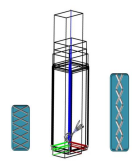

Figure 1

Acta Cryst. (2020). A76, a90 